\title{
ALTERED THERMOREGULATION IN THE IGUANA DIPSOSAURUS DORSALIS FOLLOWING EXERCISE
}

\author{
J. G. CAnnon* and M. J. Kluger \\ Department of Physiology, The University of Michigan Medical School, Ann Arbor, MI 48109, U.S.A.
}

(Received 4 April 1984; accepted 16 May 1984)

\begin{abstract}
Seven desert iguanas ran on a motorized treadmill for 20-min periods. Before, during and after exercise, the iguanas were in a thermal gradient which allowed them to thermoregulate behaviourally.

2. For several hours following exercise, the iguanas selected warmer ambient temperatures, resulting in small, but statistically significant, increases in body temperature.

3. The increases in temperature were proportional to the exercise intensity.

4. These changes were not observed if exercise was preceded by administration of the antipyretic drug. sodium salicylate.

5. These data support the hypothesis that exercise causes a change in central thermoregulatory control which may be similar to fever caused by infection.
\end{abstract}

Key Word Index - Thermoregulation; desert iguanas; Dipsosaurus dorsalis; exercise; fever; hyperthermia.

\section{INTRODUCTION}

During exercise, mammalian core temperature can increase by several degrees Celsius in a manner proportional to the exercise intensity and relatively independent of ambient temperature (Nielsen 1938). Many physiologists have hypothesized that this occurs because exercise somehow causes a resetting of the thermoregulatory "set-point" (Brooks et al., 1971; Hammel et al., 1963; Jequier, 1970; Myers et al., 1977). This hypothesis has been repeatedly tested by comparing body temperature thresholds at which effector responses (such as sweating and peripheral vasodilation) are initiated during exercise with other forms of hyperthermia. Much (Nadel, 1977; Wyndham, 1973), but not all (Johnson and Park, 1981), of the data from these experiments favour the viewpoint that no change in the thermoregulatory set-point actually occurs during exercise.

Thermoregulation after exercise has not been extensively studied. In 1973, Haight and Keatinge found that human core temperature remained elevated for many hours following long-duration exercise. Furthermore, sweat responses to heat and metabolic responses to cold occurred at warmer temperatures after exercise implying that the setpoints of these subjects were elevated. The authors suggested that endogenous pyrogen (EP), the mediator of fever and other non-specific host defence responses during infection, may be responsible for these results. Support for this theory comes from a recent investigation showing that a factor is present in human plasma after exercise which causes fever and other responses associated with EP (interleukin 1) when injected into rats (Cannon and Kluger, 1983).

*Present address: Department of Medicine, Tufts University School of Medicine, 136 Harrison Avenue, Boston, MA 02111 , U.S.A.
In exercising mammals, the high rates of heat production and heat elimination may make detection of a change in the thermoregulatory set-point following exericise difficult. Other consequences of exercise, such as fluid shifts and dehydration, sometimes compromise heat dissipation mechanism (Greenleaf and Castle, 1971; Nielsen et al,, 1971) and thus lead to an apparent, but not actual, shift in the set-point.

A poikilotherm such as the desert iguana Dipsosaurus dorsalis may be a better animal model to study changes in the thermoregulatory set-point since it does not produce or store significant amounts of metabolic heat. This animal depends upon environmental heat sources to regulate body temperature. Thus, the body temperature attained through behavioural selection of a preferred ambient temperature reflects the status of the lizard's thermoregulatory system (Bernheim and Kluger, 1976, 1977) and is not complicated by fluid losses from sweating, internal fluid compartment shifts or changes in energy substrate availability. A large body of evidence has shown that the primary effector mechanism of poikilotherms is behavioural selection of an appropriate ambient environment. Thus, an increase in the body temperature of a free-ranging lizard in a steady thermal gradient represents a repositioning of the animal in a warmer part of the gradient.

In the present study, 7 desert iguanas were exercised on a monitorized treadmill. Cloacal temperature was monitored before, during and after exercise to determine if changes in thermoregulation occur compared to time control periods. Two treadmill speeds were employed to ascertain if observed elevations in body temperature correlated with exercise intensity or were merely non-specific stress responses to handling and the novelty of the protocol. Finally, to test the hypothesis that observed increases in body temperature following exercise may be mediated by a mechanism similar to that causing fever 
during infection, the antipyretic drug sodium salicylate was administered before exercise in an attempt to block the rise in body temperature.

\section{MATERIALS AND METHODS}

Seven desert iguanas, mean body wt $70 \pm 5 \mathrm{~g}$ (SEM), were maintained in individual $0.2 \mathrm{~m}$ high $\times 0.2 \mathrm{~m}$ wide $\times 1.2 \mathrm{~m}$ long enclosures with sandcovered floors. The enclosures were illuminated between $6 \mathrm{a} . \mathrm{m}$. and $6 \mathrm{p} . \mathrm{m}$. with fluorescent lights. Between 8:30 a.m. and 4:30 p.m., $250 \mathrm{~W}$ heat lamps at one end of each enclosure created a thermal gradient ranging from $55^{\circ} \mathrm{C}$ under the lamps to $25^{\circ} \mathrm{C}$ at the other end. When all lamps were off, ambient temperature was a uniform $22 \pm 2 \mathrm{C}$. The animals were fed canned dog food, romaine lettuce, dandelion flowers and bananas.

A custom-built treadmill provided a $0.2 \times 0.6 \mathrm{~m}$ running surface and continuously variable speed control up to $2.5 \mathrm{~km} \mathrm{~h}^{-1}$. The running surface was surrounded with $0.2 \mathrm{~m}$ high cardboard walls and one corner was irradiated with a $250 \mathrm{~W}$ heat lamp. The animals were kept moving by lightly stroking their tails against the grain of the skin or gently tapping the upper posterior hindlimb area. Preliminary experiments indicated that the lizards could maintain a pace of $0.65 \mathrm{~km} \mathrm{~h}^{-1}$ for $20 \mathrm{~min}$ which is similar to the exercise performance of $D$. dorsalis reported by others (John-Alder and Bennett, 1981). At higher speeds, respiratory movements became exaggerated. The animals would soon lock their legs, expand their thorax and abdomen and close their eyelids. Once adopting this defensive posture, the animals could not be coaxed into further movement.

A copper-constantan thermocouple sheathed in PE- 160 polyethylene tubing was inserted $c a .5 \mathrm{~cm}$ into the cloaca and taped to the tail of each lizard at 8 a.m. Temperature was recorded at $30-\mathrm{s}$ intervals on a Honeywell Electronik 112 multipoint recorder.

Experiment 1. Responses of lizards to low-and highspeed exercise

Each animal exercised on the treadmill for $20 \mathrm{~min}$ at $0.54 \mathrm{~km} \mathrm{~h}^{-1}$ (low speed) or at $0.65 \mathrm{~km} \mathrm{~h}^{-1}$ (high speed) and was then returned to its thermal gradient enclosure. On a given day, no more than 3 lizards were subjected to any exercise protocol and only one animal was placed on the treadmill at a time. Exercise periods began at 9:30, 9:50 and 10:10 a.m.

\section{Experiment 2. Time controls}

Two time controls were used. Undisturbed time controls consisted of 7 lizards which were immediately returned to the thermal gradient enclosures after insertion of the cloacal thermocouples and left undisturbed. A second time control group consisted of 7 lizards placed on the exercise treadmill, but not exercised. This control group was necessary since in Experiment 1 it was found that the lizards lost a great deal of heat while exercising on the treadmill (see Results). To ensure that the post-exercise temperature elevation was not merely a compensatory response to this heat loss while exercising, lizards were placed on the stationary treadmill for $20 \mathrm{~min}$ with the heat lamp off. The animals were handled and prodded form time to time to simulate the exercise protocols as much as possible. At the end of this period, the lizards were returned to the thermal gradient and left undisturbed.

Experiment 3. Effect of sodium salicylate on body temperature of rested and exercised lizards

At 9:30 a.m., groups of lizards (less than 4 on any given day) were given an i.p. injection of $7.5 \mathrm{mg}$ sodium salicylate in sodium chloride solution (saline). This dose of sodium salicylate has been shown to block fever in infected lizards (Bernheim and Kluger. 1976). The animals were then either exercised at $0.54 \mathrm{~km} \mathrm{~h}^{-1}$ for $20 \mathrm{~min}$ and then returned to their thermal gradient, or not exercised (controls) and returned to their thermal-gradients. For the next several hours, body temperature was monitored in these groups of undisturbed lizards.

Statistical comparisons were made by analysis of variance. Significant differences between any two groups were then determined by Tukey's method for equal sample sizes.

\section{RESULTS}

Experiment 1. Responses of lizards to low-and highspeed exercise

Figure 1 illustrates the temperature variations of two animals exercised for $20 \mathrm{~min}$ at $0.54 \mathrm{~km} \mathrm{~h}^{-1}$ compared to the same animals when they were undisturbed. The cloacal temperature of the lizards oscillated over a range of several degrees as the animals shuttled between warmer and cooler parts of the thermal gradient. When hourly averages were computed, however, these rapid temperature fluctuations were damped out, illustrating a tightly regulated circadian pattern which peaked at 10-11 a.m. and slowly declined over the next several hours (Fig. 2,

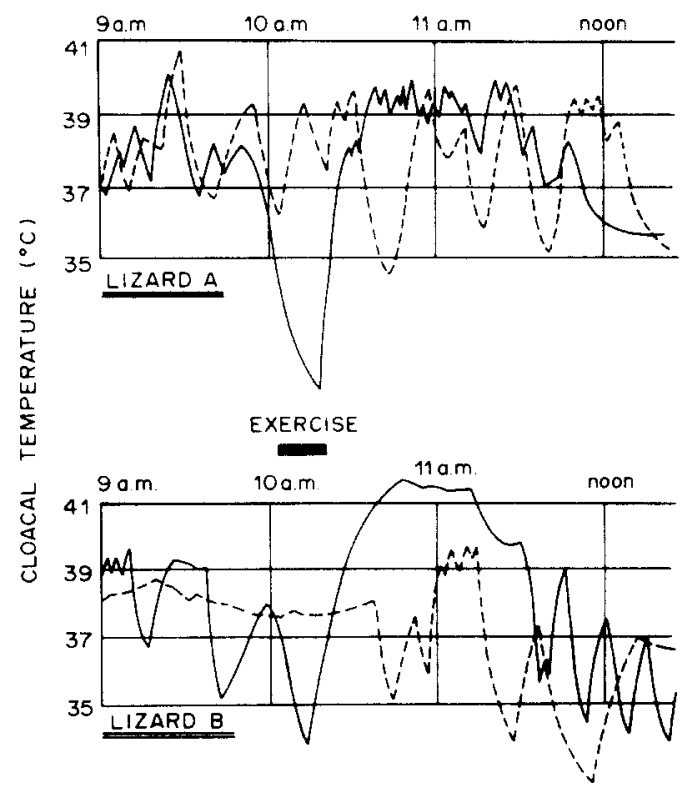

Fig. 1. The individual responses of 2 lizards to $20 \mathrm{~min}$ of exercise at $0.54 \mathrm{~km} \mathrm{~h}^{-1}$ ( - ) are compared to undisturbed thermoregulatory behaviour (-- -). 


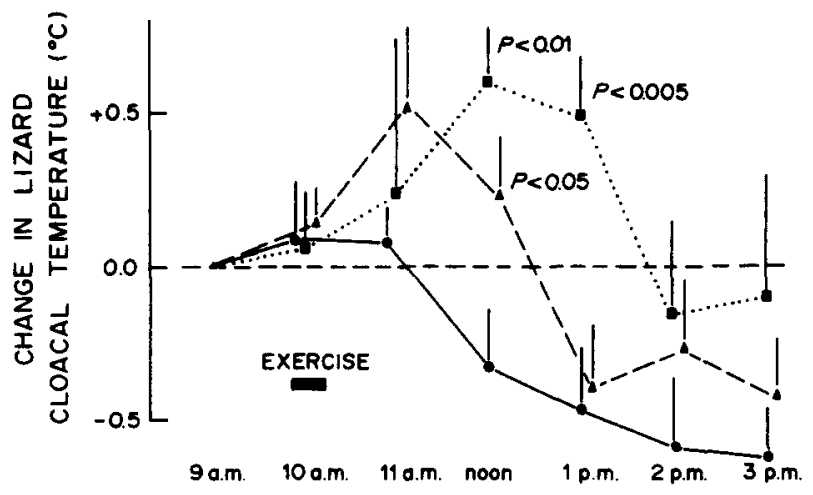

Fig. 2. The change in lizard cloacal temperature from 9 a.m. baseline (hourly means $\pm \mathrm{SEM}, n=7$ ). The following interventions were performed at approx. 10 a.m.: (i) $O$, undisturbed in thermal gradient enclosures, body temperature $\left(T_{\mathrm{b}}\right)$ at $9 \mathrm{a} \cdot \mathrm{m} .=37.8 \pm 0.2^{\circ} \mathrm{C}$; (ii) $\Delta$, exercise at $0.54 \mathrm{~km} \mathrm{~h}^{-1}$ for $20 \mathrm{~min}, T_{\mathrm{b}}$ at $9 \mathrm{a} . \mathrm{m} .=37.6 \pm 0.3^{\circ} \mathrm{C}$; (iii) $\mathrm{G}$, exercise at $0.65 \mathrm{~km} \mathrm{~h}^{-1}$ for $20 \mathrm{~min}, T_{\mathrm{b}}$ at $9 \mathrm{a} . \mathrm{m} .=37.3 \pm 0.3^{\circ} \mathrm{C}$.

The cloacal temperature of the lizards dropped progressively during the 20 min exercise period even though a heat source was available to them (Fig. 1, 10 a.m.). Apparently, keeping pace with the treadmill and avoiding the prodding finger of the experimenter took precedence over short-term thermoreguiation. The precipitous fall in temperature appeared to be an artefact of the protocol, therefore, the temperature data collected during the $20 \mathrm{~min}$ on the treadmill was not included in the time-averaged data which follows.

After exercise, the lizards behaviourally regulated at higher temperatures. Figure 1 presents examples of the two strategies employed by the lizards to elevate their temperature. Lizard A spent brief periods in the warm end of the gradient, but returned there often, resulting in more rapid oscillations of body temperature. In this case maximum temperatures did not exceed those observed during control periods, however minimum temperatures did not fall as low as during control periods, resulting in a higher average body temperature. Lizard B, on the other hand, moved to the warmer end of the gradient and remained there, resulting in a dramatically higher maximum temperature over control measurements.

The temperature elevation after exercise is more readily discernable when the data are plotted as time-averaged changes from baselines as in Fig. 2. Following low intensity exercise, the cloacal temperatures of the lizards as a group was significantly elevated over control $(P<0.05)$ approx. $90 \mathrm{~min}$ after exercise. The elevation in cloacal temperature after higher intensity exercise was of greater magnitude $\left(0.9^{\circ} \mathrm{C}, P<0.01\right)$ and duration. The correlation between running speed (either $0,0.55$ and $0.65 \mathrm{~km} \mathrm{~h}^{-1}$ ) and mean $3 \mathrm{~h}$ change in temperature was $0.55(P=0.01)$.

\section{Experiment 2. Time controls}

Cloacal temperature fell progressively while the lizards were on the motionless treadmill without access to the heat lamp. This procedure led to erratic thermoregulatory behaviour when the animals were returned to their thermal gradients. However, this handling, introduction to a novel environment, and cold stress did not cause the lizards to increase their temperature over undisturbed control values for the
$3 \mathrm{~h}$ period in which post-exercise responses were recorded (Fig. 3).

Experiment 3. Effect of sodium salicylate on body temperature selection of rested and exercised lizards

Administration of sodium salicylate did not significantly alter the thermoregulatory patterns of non-exercised (rested) lizards. The $3 \mathrm{~h}$ average temperature after injection of sodium salicylate was slightly, but not statistically significantly, higher than during the same period without injection of sodium salicylate (see left-hand side of Fig. 4). Sodium salicylate administered immediately before exercise completely abolished the post-exercise rise in body temperature, and actually caused a suppression of body temperature for at least $3 \mathrm{~h}$ following exercise (right-hand side of Fig. 4).

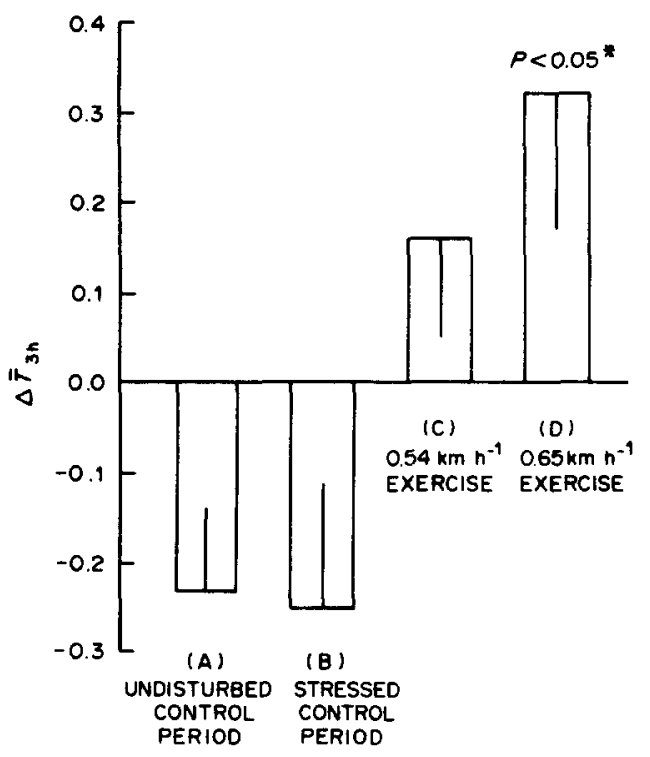

Fig. 3. Average change in cloacal temperature for $3 \mathrm{~h}$ following: (A) lizards left undisturbed in the thermal gradient chamber; (B) lizards placed on the treadmill, but not exercised; (C) lizards exercised for $20 \mathrm{~min}$ at $0.54 \mathrm{~km} \mathrm{~h}^{-1}$; and (D) lizards exercised for $20 \mathrm{~min}$ at $0.65 \mathrm{k} \mathrm{h}^{-1}$. Data are normalized to 9 a.m. baseline temperatures. *Significant different from (A) and (B). 


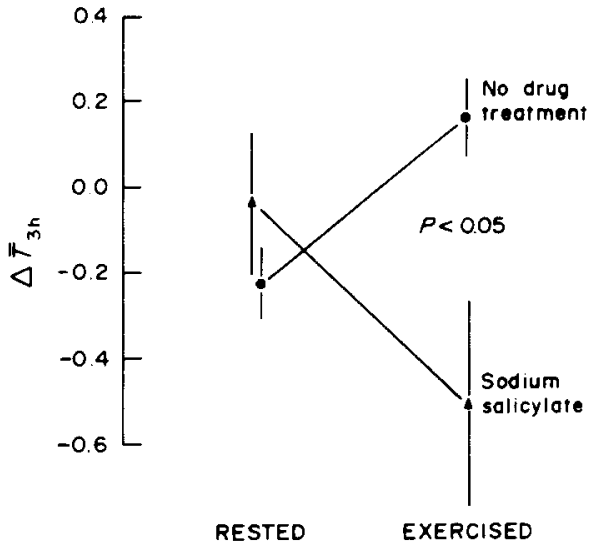

Fig. 4. Three-hour average change in lizard cloacal temperature $(n=7)$ after control or exercise periods with and without prior i.p. injection of $7.5 \mathrm{mg}$ sodium salicylate.

\section{DISCUSSION}

These experiments show that the desert iguana responds to treadmill exercise with a gradual rise in body temperature which reaches a maximum elevation approx. 90-120 min following activity. It must be emphasized that $D$. dorsalis thermoregulates almost exclusively by behavioural means (DeWitt, 1967). Therefore, this temperature elevation after exercise is clearly not a consequence of impaired effector mechanisms for heat dissipation; the animal must actively seek out a warmer environment. Furthermore, as indicated by the fall in temperature during exercise (Fig. 1), the lizards actually lost more heat during exercise (due to the inability to maintain proper position under the heat lamp) than they produced (through increased metabolic rate). Therefore, the ensuing temperature elevation cannot be explained as the load-error in a negative feedback control system.

This series of experiments represents a trade-off of one set of uncertainties for another. While this poikilothermic model is advantageous because it circumvents complications of effector origin, it is possible that the lizard's behaviour may be influenced by a variety of factors and not the exercise per se. However, since the rise observed after exercise was proportional to the exercise intensity and not evoked by handling and environmental stresses alone, this concern is diminished. Further, it is not likely that thermoregulation after exercise was impaired either through fatigue or some hyperkinetic state since no common pattern was observed; some animals were very active, others were not. The only common facet of their behaviour was that it resulted in an elevated averaged body temperature.

The time-course of the rise in body temperature and blockade by the drug sodium salicylate are similar to the development of a fever and antipyresis, respectively (Bernheim and Kluger, 1976). Exercising human subjects release an endogenous pyrogen-like substance into their plasma (Cannon and Kluger, 1983) and it is possible that exercising lizards release a similar pyrogen (Bernheim and Kluger, 1977). Although the dose of sodium salicylate used in these experiments did not affect body temperature of nonexercised lizards, this dose did affect thermoregulation after exercise. It is, however, possible that the salicylate was not "blocking a fever" since body temperature actually fell after the low-intensity exercise and the lizards were not able to perform the high-intensity exercise after salicylate treatment. The drug may have affected acid-base balance or some aspect of arachidonate metabolism which only indirectly influenced temperature regulation.

While these behavioural and pharmacological results must be viewed with some caution, they do support the "elevated set-point hypothesis" suggested for mammals. The fact that a post-exercise "hyperthermia" can be elicited in an animal which exhibited a net loss of heat during exercise argues that a change in central thermoregulatory drive has occurred rather than a change in peripheral thermoregulatory capability.

Acknowledgements - We thank Orin Gelderloos for loaning us the lizards, John Faulkner and Timothy White for use of the small animal treadmill and Jeffrey Tatro and Wendy Scales for critically reviewing the manuscript.

This work was supported by NIH Grant AI 13878.

\section{REFERENCES}

Bernheim H. A. and Kluger M. J. (1976) Fever and antipyresis in the lizard Dipsosaurus dorsalis. Am. J. Physiol. 231, 198-203.

Bernheim H. A. and Kluger M. J. (1977) Endogenous pyrogen-like substance produced by reptiles. $J$. Physiol., Lond. 267, 659-666.

Brooks G. A.. Hittelman K. J., Faulkner J. A. and Beyer B. E. (1971) Tissue temperatures and whole-animal oxygen consumption after exercise. Am. J. Physiol. 221, 427-431.

Cannon J. G. and Kluger M. J. (1983) Endogenous pyrogen activity in human plasma after exercise. Science 220, 617-619.

DeWitt C. B. (1967) Precision of thermoregulation and its relation to environmental factors in the desert iguana, Dipsosaurus dorsalis. Physiol. Zool. 40, 49-66.

Greenleaf J. E. and Castle B. L. (1971) Exercise temperature regulation in man during hypohydration and hyperhydration. J. appl. Physiol. 30, 847-853.

Haight J. S. J. and Keatinge W. R. (1973) Elevation in set point for body temperature regulation after prolonged exercise. J. Physiol., Lond. 229, 77-85.

Hammel H. T., Jackson D. C.. Stolwijk J. A. J., Hardy J. D. and Stromme S. B. (1963) Temperature regulation by hypothalamic proportional control with an adjustable set point. J. appl. Physiol. 18, 1146-1154.

Jequier E. (1970) Reduced hypothalamic set point temperature during exercise in man. Experientia 26, 681.

John-Alder H. B. and Bennett A. F. (1981) Thermal dependence of endurance and locomotory energetics in a lizard. Am. J. Physiol. 241, R342-R349.

Johnson J. M. and Park M. K. (1981) Effect of upright exercise on threshold for cutaneous vasodilation and sweating. J. appl. Physiol. 50, 814-818.

Myers R. D. Gisolfi $\dot{C}$. V. and Mora F. (1977) Calcium levels in the brain underlie temperature control during exercise in the primate. Nature 266, 178-179.

Nadel E. R. (1977) Problems with Temperature Regulation During Exercise, pp. 1-141. Academic Press, New York. Nielsen B., Hansen G., Jorgensen S. O. and Nielsen E. (1971) Thermoregulation in exercising man during dehy- 
dration and hyperhydration with water and saline. Int. J. Tukey J. W. (1949) Comparing individual means in the Biomet. 15, 195-200.

analysis of variance. Biometrics $5,99-114$.

Nielsen M. (1938) Die regulation der korpertemperatur bei Wyndham C. H. (1973) The physiology of exercise under muskelarbeit. Skand. Arch. Physiol. 79, 193-230. heat stress. A. Ret. Physiol. 35, 193-220. 